\title{
Surveillance for Hepatocellular Carcinoma Also Improves Survival of Incidentally Detected Intrahepatic Cholangiocarcinoma Arisen in Liver Cirrhosis
}

\author{
Francesco Tovolia $^{a}$ Pietro Guerra ${ }^{a} \quad$ Massimo lavarone ${ }^{b} \quad$ Letizia Veronese $^{c}$ \\ Matteo Renzulli $^{d}$ Stefania De Lorenzo ${ }^{e}$ Francesca Benevento $^{a}$

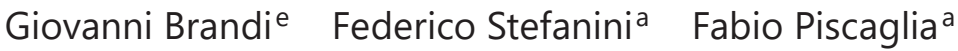 \\ ${ }^{a}$ Division of Internal Medicine, Azienda Ospedaliero-Universitaria di Bologna, Bologna, Italy; \\ b Foundation IRCCS Ca' Granda Ospedale Maggiore Policlinico, Division of Gastroenterology \\ and Hepatology, Milan, Italy; ' III Medical Clinic, Department of Internal Medicine,

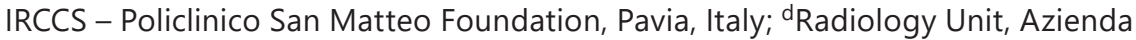 \\ Ospedaliero Universitaria S.Orsola-Malpighi di Bologna, Bologna, Italy; ${ }^{e}$ Oncologia Medica, \\ Azienda Ospedaliero-Universitaria di Bologna, Bologna, Italy
}

\author{
Keywords \\ Cholangiocellular carcinoma - Intrahepatic cholangiocarcinoma - Screening · Outcomes · \\ Liver cirrhosis
}

\begin{abstract}
Background: Due to its poor survival, intrahepatic cholangiocarcinoma (ICC) is held to be a much more aggressive cancer than hepatocellular carcinoma (HCC). In most published series, patients were diagnosed when symptomatic. However, ICC is now increasingly being discovered during the surveillance for HCC in cirrhosis. Whether this earlier detection of ICC is associated with an equally dismal prognosis or not is unknown. Methods: This is amulticenter retrospective study of consecutive ICC patients. Patients were stratified into subgroups according to the absence/presence of cirrhosis. A propensity score matching was performed to reduce the potential biases. Cirrhotic patients were further stratified according to their surveillance status. The lead-time bias and its potential effects were also estimated. Results: We gathered 184 patients. Eighty-five patients (46.2\%) were cirrhotic. Liver cirrhosis was not related to a worse overall survival (33.0 vs. 32.0 months, $p=0.800$ ) even after the propensity score analysis ( 43.0 in vs. 44.0 months in 54 pairs of patients, $p=0.878$ ). Among the cirrhotic population, 47 (55.3\%) patients had received a diagnosis of ICC during a surveillance programme. The 2 subgroups differed in maximum tumour dimensions ( 30 vs. $48 \mathrm{~mm}$ in surveyed and non-surveyed patients, respectively). Surveyed patients were more likely to receive surgical treatments ( 59.8 vs. $28.9 \%, p=0.003$ ). Overall survival was higher in surveyed patients (51.0
\end{abstract}


vs. 21.0 months, $p<0.001$ ). These benefits were confirmed after correcting for the lead-time bias. Conclusions: Cirrhotic patients have different clinical presentation and outcomes of ICC according to their surveillance status. In our series, ICC in cirrhosis was not associated with worse OS. Cirrhosis itself should not discourage either surgical or non-surgical treatments.

(C) 2020 The Author(s)

Published by S. Karger AG, Basel

\section{Introduction}

Intrahepatic cholangiocarcinoma (ICC) is the second most frequent primary liver cancer, following hepatocellular carcinoma (HCC) [1]. Chronic liver diseases, and in particular liver cirrhosis, are a well-known risk factors for ICC [1]. However, the prognostic relevance of liver cirrhosis is still unknown. Current evidence relies on few single-centre retrospective studies, which provided contradictory results. In 2 Eastern surgical series, Li et al. [2] reported that survival rates were poorer for patients with cirrhosis than for those without cirrhosis, whereas Luo et al. [3] identified liver cirrhosis as a protective factor. In the only Western series (which included both surgical and non-surgical cases), Jesper et al. [4] reported no difference in survival between non-cirrhotic patients and patients with compensated cirrhosis. Interestingly, ICC can sometimes be discovered during the surveillance for HCC in cirrhosis [5]. None of these studies reported whether cirrhotic patients received the diagnosis of ICC in the setting of ultrasound surveillance, which is commonly recommended for the early detection of HCC and that might, theoretically, lead to an earlier diagnosis of ICC as well.

Despite this relative lack of evidence, the treatment of patients with both liver cirrhosis and ICC is gaining clinical significance due to the increasing prevalence of both conditions [6-8]. It also raises the question of whether these patients constitute a distinct group needing to be treated differently from patients without cirrhosis. Therefore, we aimed to assess the prognostic role of liver cirrhosis and the potential effects of ultrasound surveillance in a large collaborative multicenter study.

\section{Methods}

Study Design and Clinical Setting

This retrospective observational study included all patients who received a histological diagnosis of ICC in 4 centres located in 3 different institutions (S.Orsola-Malpighi Hospital - Bologna; Fondazione IRCCS Ca' Granda - Milan; Policlinico S. Matteo - Pavia) between January 2003 and December 2017. The date of the last follow up was August 31, 2019.

The following data were available for each patient: age, sex, history of underlying liver diseases and liver functioning laboratory tests, Eastern Cooperative Oncology Group Performance Status, tumour dimension, multinodularity, and spread to lymphnodes or distant organs. Tumour staging was re-categorized according to the American Joint Committee on Cancer-TNM (AJCC-TNM) eighth edition classification for ICC [9].

Assessment of Liver Cirrhosis

Patients were divided into subgroups according to the absence/presence of liver cirrhosis. Cirrhosis was diagnosed either based on histology or on a combination of non-invasive paramenters (including liverdirected physical exam, routine blood tests, elastography, liver imaging and fibrosis scores), according to the standard of practice $[10,11]$.

Definition of Ultrasound Surveillance in Cirrhotic Patients

Patients with liver cirrhosis were further distinguished in patients undergoing ultrasound surveillance for HCC and patients who had not been surveyed. ICC was considered to be diagnosed during surveillance if it was first detected during the semi-annual ultrasound surveillance programme, which is recommended for

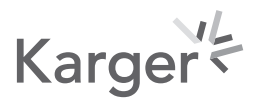


Tovoli et al.: Intrahepatic Cholangiocarcinoma in Liver Cirrhosis

cirrhotic patients for early detection of HCC. Patients who received a simultaneous diagnosis of ICC and cirrhosis or who already had a diagnosis of cirrhosis but did not perform or were not compliant with the semiannual surveillance protocol were considered non-surveyed patients.

Ethics

The Ethics Committee approved the study (protocol 78/2017/O/OSSN), which was conducted according to the ethical guidelines of the 1975 Declaration of Helsinki. Considering the retrospective design of the study and the unfavourable prognosis of the investigated disease, the Ethics Committee waived the need for informed consent for deceased patients and for patients whose clinical conditions had worsened to a point in which they were not able to sign a valid consent. All of the remaining patients signed the informed consent for this study.

\section{Statistics}

Continuous data are expressed as median (range). Categorical variables have been reported as frequencies. Group comparisons were performed with the Mann-Whitney test for continuous variables and with the Fisher test or the Cochran-Armitage test for trend, as appropriate. Overall survival (OS) was defined as the time between the diagnosis of ICC and death or last follow-up examination. Survival curves were estimated using the product-limit method of Kaplan-Meier. The role of stratification factors was analysed with log-rank tests. The role of prognostic factors was investigated with a Cox-regression analysis. Recognized prognostic factors of ICC as well as variables reaching a $p$ value $<0.10$ at the univariate analysis were included in the multivariable models. A first multivariable model was designed including only the main baseline clinical and tumour characteristics of ICC patients. This model was carried out to enable a comparison with previous studies which used similar variables. Subsequently, we designed a second multivariable model, that included also the treatment received, as it usually represents the main predictor of survival. The latter model was further used for the calculation of the propensity score. $p$ values $<0.05$ were considered statistically significant.

To limit the effects of possible selection bias, we performed a propensity score analysis to correct the imbalances of prognosticators when comparing cirrhotic and non-cirrhotic patients. The propensity score is the conditional probability of being treated given a set of observed potential confounders. In this way, all the information from a group of potential confounders is summarized into a single-balancing score variable. Propensity score assures that the distribution of measured baseline covariates is maintained unchanged across the study groups.

Lead-time bias is defined as the false impression of improved survival in a screened population without affecting mortality because the cancer is diagnosed earlier in the natural history of the disease, but the patient still dies of cancer [12]. To address the potential lead-time bias when estimating the effects of surveillance in cirrhotic patients, we calculated the lead time of ICC in the same methodological line used for other screening programmes [13-15]. To assess the doubling time of ICC, we searched the Medline database from January 2000 to August 2019 with the keywords or Medical Subject Headings "cholangiocarcinoma” AND "doubling time" AND/OR "growth rate." All the studies were limited to humans and literature in English. The assumptions for the sojourn time and the consequent procedures for calculating the lead time are reported in the Appendix. Statistical analyses were performed using SPSS version 23.0 (SPSS Inc., Chicago, IL, USA).

\section{Results}

\section{Characteristics of the Whole Study Population}

A total of 185 patients were included in this study (Table 1). Female gender was slightly predominant (57.3\%), and the median age at the diagnosis of ICC was 65 years. Sixteen patients (8.7\%) had an underlying hepatitis B virus infection, 38 (20.7\%) a chronic hepatitis $\mathrm{C}$ virus infection, 48 (26.1\%) a non-viral (mainly alcoholic or dysmetabolic) chronic liver disease, while 82 patients (44.6\%) had no evident underlying liver diseases. Solitary tumours were found in 123 (66.5\%) patients, with a median diameter of $55 \mathrm{~mm}$ (IQR 35-90). Nodal involvement was found in 29 (15.8\%) patients and distant metastasis in 19 patients $(10.3 \%)$.

\section{Karger ${ }^{\prime \prime}$}




\section{Liver Cancer}

Fig. 1. OS stratified according to the TNM stage in the whole study population. OS, overall survival.

Table 1. Whole study population background

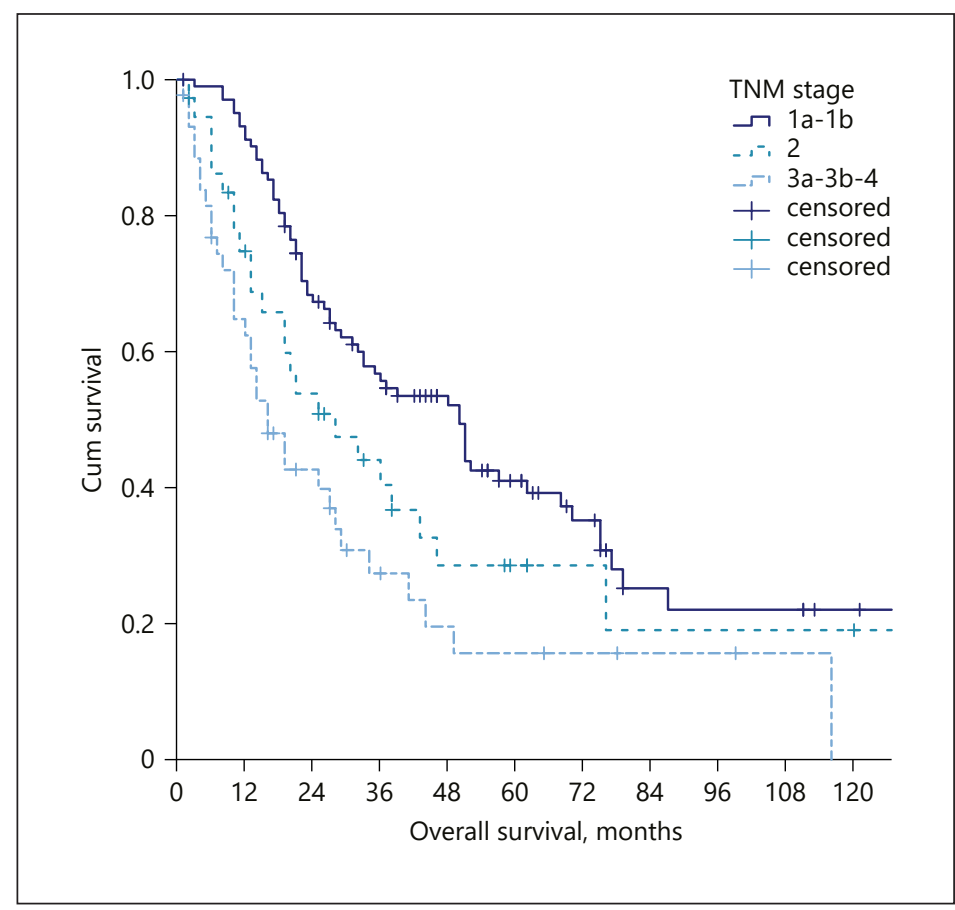

\begin{tabular}{lc}
\hline Variable & \\
\hline Age, years & $65(59-72)$ \\
Female sex & $106(57.3)$ \\
Cirrhosis & $85(46.2)$ \\
PS > & $51(27.7)$ \\
Main tumour dimension, mm & $55(35-90)$ \\
Multinodular disease & $62(33.7)$ \\
N1 & $29(15.8)$ \\
M1 & $19(10.3)$ \\
Macrovascular invasion & $14(7.6)$ \\
Tumour grading & $78(42.4)$ \\
$\quad$ G1 & $74(40.2)$ \\
G2 & $32(7.4)$ \\
G3 & \\
TNM stage & $68(37.0)$ \\
1a & $35(19.0)$ \\
1b & $37(20.1)$ \\
2 & $25(13.5)$ \\
3 & $19(10.3)$ \\
4 & $107(58.2)$ \\
Treatment & $31(16.8)$ \\
Surgery & $35(19.0)$ \\
Locoregional procedures & $11(6.0)$ \\
Chemotherapy & \\
BSC & \\
\hline
\end{tabular}

Continuous variables are expressed as median (interquartile range). Categorical variables are reported as absolute frequencies (percentage). 
Table 2. Predictors of survival in the whole study population according to the Cox regression analysis (including treatment received)

\begin{tabular}{|c|c|c|c|c|c|c|}
\hline \multirow[t]{2}{*}{ Variable } & \multicolumn{3}{|c|}{ Univariate analysis } & \multicolumn{3}{|c|}{ Multivariable analysis } \\
\hline & HR & $95 \% \mathrm{CI}$ & $p$ value & HR & $95 \% \mathrm{CI}$ & $p$ value \\
\hline Age, year & 1.004 & $0.986-1.023$ & 0.654 & & & \\
\hline Male sex & 0.738 & $0.511-1.065$ & 0.105 & & & \\
\hline \multicolumn{7}{|l|}{ Aetiology } \\
\hline Healthy liver & - & Reference & - & & & \\
\hline HBV infection & 0.625 & $0.306-1.274$ & 0.196 & & & \\
\hline HCV infection & 1.088 & $0.698-1.697$ & 0.710 & & & \\
\hline Non-viral liver disease & 0.783 & $0.495-1.241$ & 0.298 & & & \\
\hline Cirrhosis & 0.955 & $0.667-1.367$ & 0.802 & & & \\
\hline ECOG PS $>0$ & 2.036 & $1.398-2.966$ & $<0.001$ & 1.137 & $0.731-1.769$ & 0.568 \\
\hline Main tumour dimension, mm & 1.009 & $1.005-1.014$ & $<0.001$ & 1.003 & 0.998-1.009 & 0.227 \\
\hline Multinodular disease & 2.304 & $1.590-3.339$ & $<0.001$ & 1.832 & $1.203-2.789$ & 0.005 \\
\hline N1 & 1.228 & $0.743-2.028$ & 0.423 & & & \\
\hline M1 & 3.839 & $2.195-6.714$ & $<0.001$ & 1.804 & $0.912-3.596$ & 0.090 \\
\hline \multicolumn{7}{|l|}{ Tumour grading } \\
\hline G1 & - & Reference & - & - & Reference & - \\
\hline G2 & 1.446 & $0.965-2.166$ & 0.074 & 1.456 & $0.943-2.249$ & 0.090 \\
\hline G3 & 2.141 & $1.299-3.527$ & 0.003 & 1.533 & $0.900-2.611$ & 0.116 \\
\hline \multicolumn{7}{|l|}{ Treatment } \\
\hline Surgery & - & Reference & - & - & Reference & - \\
\hline Locoregional procedures & 2.005 & $1.231-3.265$ & 0.005 & 2.201 & $1.322-3.665$ & 0.002 \\
\hline Chemotherapy & 6.062 & $3.710-9.904$ & $<0.001$ & 3.211 & $1.719-6.000$ & $<0.001$ \\
\hline BSC & 16.585 & $7.696-35.742$ & $<0.001$ & 13.889 & $5.887-32.766$ & $<0.001$ \\
\hline
\end{tabular}

ECOG PS, Eastern Cooperative Oncology Group Performance Status.

Fig. 2. OS stratified according to the treatment received in the whole study population. OS, overall survival.

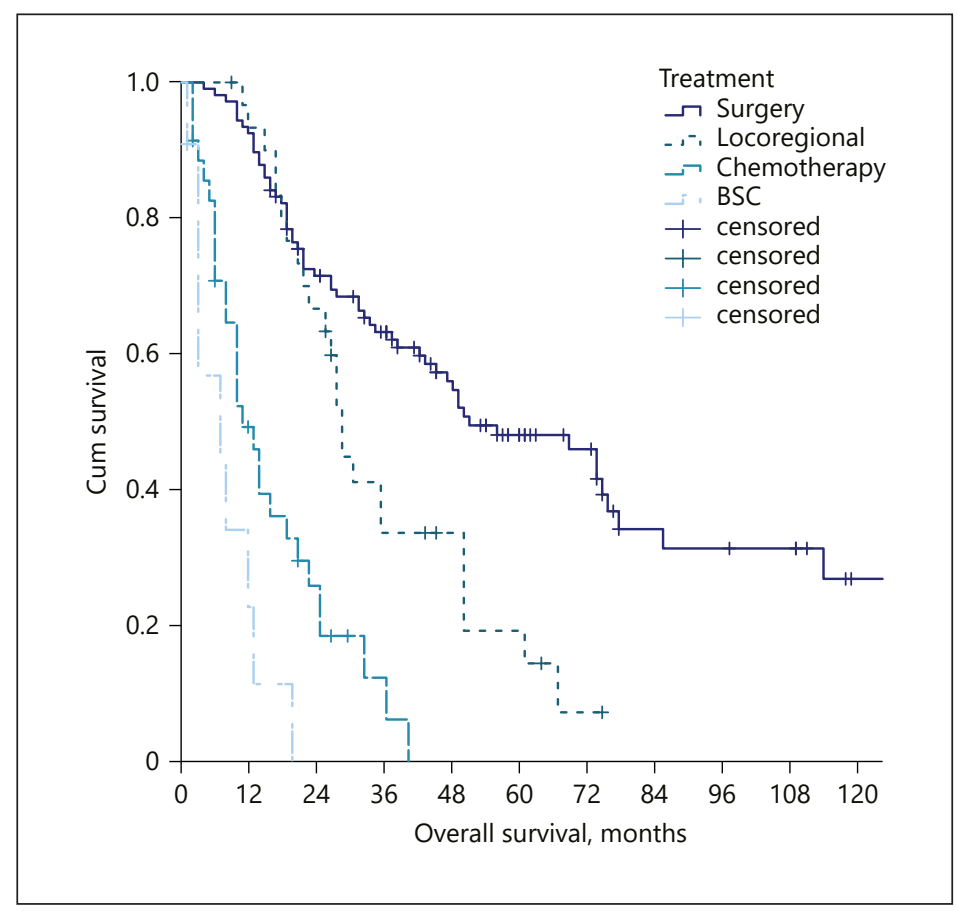


Table 3. Characteristics of patients with and without liver cirrhosis

\begin{tabular}{|c|c|c|c|}
\hline Variable & $\begin{array}{l}\text { Non-cirrhotic patients } \\
(n=99)\end{array}$ & $\begin{array}{l}\text { Cirrhotic patients } \\
(n=85)\end{array}$ & $p$ value \\
\hline Age, years & $65(57-72)$ & $65(60-72)$ & 0.872 \\
\hline Female sex & $53(53.5)$ & $26(30.6)$ & 0.002 \\
\hline $\mathrm{PS}>0$ & $71(71.7)$ & $62(72.9)$ & 0.870 \\
\hline Main tumour dimension, mm & $80(50-100)$ & $36(23-58)$ & $<0.001$ \\
\hline Multinodular disease & $39(39.4)$ & $23(27.1)$ & 0.087 \\
\hline N1 & $21(21.2)$ & $8(9.4)$ & 0.041 \\
\hline M1 & $15(15.2)$ & $4(4.7)$ & 0.027 \\
\hline Macrovascular invasion & $4(4.0)$ & $10(11.8)$ & 0.056 \\
\hline \multicolumn{4}{|l|}{ Tumour grading } \\
\hline G1 & $36(36.4)$ & $42(49.4)$ & \multirow[t]{3}{*}{0.338} \\
\hline G2 & $47(47.5)$ & $27(31.8)$ & \\
\hline G3 & $16(16.2)$ & $16(18.8)$ & \\
\hline \multicolumn{4}{|l|}{ TNM stage } \\
\hline $1 \mathrm{a}$ & $20(20.2)$ & $48(56.5)$ & \multirow[t]{6}{*}{$<0.001$} \\
\hline $1 b$ & $28(28.3)$ & $7(8.2)$ & \\
\hline 2 & $21(21.2)$ & $16(18.8)$ & \\
\hline $3 a$ & 0 & $1(1.2)$ & \\
\hline $3 b$ & $15(15.2)$ & $9(10.6)$ & \\
\hline 4 & $15(15.2)$ & $4(4.7)$ & \\
\hline \multicolumn{4}{|l|}{ Treatment } \\
\hline Surgery & $68(68.7)$ & $39(45.9)$ & 0.003 \\
\hline Locoregional procedures & $1(1.0)$ & $30(35.3)$ & $<0.001$ \\
\hline Chemotherapy & $24(24.2)$ & $11(12.9)$ & 0.060 \\
\hline BSC & $6(6.1)$ & $5(5.9)$ & 1.000 \\
\hline
\end{tabular}

Continuous variables are expressed as median (interquartile range). Categorical variables are reported as absolute frequencies (percentage).

Staging according to the AJCC TNM classification was as follows: stage 1: $56 \%$; stage 2: $20.1 \%$, stages 3-4: 23.9\%. A majority of patients underwent surgery (58.2\%). Locoregional procedures (16.8\%), systemic chemotherapy (19.6\%), or best supportive care $(6.0 \%)$ were utilized as treatments in the remaining patients. At the end of the follow-up period, $120(65.2 \%)$ patients had died. The median overall survival was 33.0 months (95\% CI 25.0-41.0). The stratification according to the AJCC-TNM classification identified different survivals according to the tumour stage (Fig. 1). Also, survival differed according to the received treatment, with a median OS of 52.0 months for surgery, 29.0 months for locoregional treatments, 11.0 for chemotherapy, and 7.0 months for best supportive care (Fig. 2).

At the Cox univariate analysis, tumour dimensions, multinodularity, distant metastasis, performance status, and tumour grading were related to the overall survival. The first multivariable model confirmed tumour dimensions, multinodularity, distant metastasis, and performance status as independent predictors of survival (online suppl. Table 1; for all online suppl. material, see www.karger.com/doi/10.1159/000509059). In the second model, that included also the treatment received, the multivariate analysis confirmed treatment and multinodularity as the sole predictors of survival (Table 2).

Comparison between Cirrhotic and Non-Cirrhotic Patients

Eighty-five patients (46.2\%) of the whole population were cirrhotic. The Child-Pugh class was A5 in 65 (76.5\%), A6 in 12 (14.1\%), B7 in 6 (7.1\%), and B8 in 2 patients (2.4\%). 
Table 4. Characteristics of cirrhotic patients according to their surveillance status

\begin{tabular}{|c|c|c|c|}
\hline Variable & $\begin{array}{l}\text { Non-surveilled patients } \\
(n=38)\end{array}$ & $\begin{array}{l}\text { Surveilled patients } \\
(n=47)\end{array}$ & $p$ value \\
\hline Age, years & $68(60-74)$ & $63(59-70)$ & 0.064 \\
\hline Female sex & $11(28.9)$ & $15(31.9)$ & 0.816 \\
\hline $\mathrm{PS}>0$ & $12(31.6)$ & $11(23.4)$ & 0.466 \\
\hline \multicolumn{4}{|l|}{ Child-Pugh class } \\
\hline A5 & $29(76.3)$ & 37 (78.7) & \multirow[t]{3}{*}{0.643} \\
\hline A6 & 5 (13.2) & 7 (14.9) & \\
\hline B7-B8 & $4(10.5)$ & $3(6.4)$ & \\
\hline Main tumour dimension, $\mathrm{mm}$ & $48(35-80)$ & $30(17.42)$ & $<0.001$ \\
\hline Multinodular disease & $13(34.2)$ & $10(21.3)$ & 0.223 \\
\hline N1 & 5 (12.3) & $3(6.4)$ & 0.458 \\
\hline M1 & $3(7.9)$ & $1(2.1)$ & 0.320 \\
\hline Macrovascular invasion & $2(5.3)$ & $8(17.0)$ & 0.174 \\
\hline \multicolumn{4}{|l|}{ Tumour grading } \\
\hline G1 & $13(34.2)$ & $29(61.7)$ & \multirow[t]{3}{*}{0.015} \\
\hline G2 & $15(39.5)$ & $12(25.5)$ & \\
\hline G3 & $10(26.3)$ & $6(12.8)$ & \\
\hline \multicolumn{4}{|l|}{ TNM stage } \\
\hline $1 \mathrm{a}$ & $16(42.1)$ & $32(68.1)$ & \multirow[t]{6}{*}{0.037} \\
\hline $1 \mathrm{~b}$ & $6(15.8)$ & $1(2.1)$ & \\
\hline 2 & $7(18.4)$ & $9(19.1)$ & \\
\hline $3 a$ & 0 & $1(2.1)$ & \\
\hline $3 b$ & $6(15.8)$ & $3(6.4)$ & \\
\hline 4 & $3(7.9)$ & $1(2.1)$ & \\
\hline \multicolumn{4}{|l|}{ Treatment } \\
\hline Surgery & $11(28.9)$ & $28(59.6)$ & 0.008 \\
\hline Locoregional procedures & $16(42.1)$ & $14(29.8)$ & 0.262 \\
\hline Chemotherapy & $6(15.8)$ & 5 (10.6) & 0.530 \\
\hline BSC & $5(13.2)$ & 0 & 0.056 \\
\hline
\end{tabular}

Continuous variables are expressed as median (interquartile range). Categorical variables are reported as absolute frequencies (percentage).

Compared to the non-cirrhotic cohort, cirrhotic patients were more often males (53.5 vs. $30.6 \%$ ), had smaller tumours at the diagnosis (36 vs. $80 \mathrm{~mm}$ ), and a rarer occurrence of nodal involvement (9.4 vs. $21.2 \%$ ) and distant metastasis (4.7 vs. 15.2\%) (Table 3). Nonetheless, cirrhotic patients were less likely to undergo surgery (45.9 vs. $68.7 \%$ ) but received locoregional treatments more often (35.3 vs. 1.0\%). There was also a trend for a reduced likelihood of receiving chemotherapy ( 12.9 vs. $24.2 \%, p=0.060)$. On the contrary, the rate of patients receiving best supportive care was similar in the 2 groups (Table 3 ). The crude survival did not differ from that of non-cirrhotic patients (33.0 vs. 32.0 months, $p=0.800$ ).

The propensity score analysis identified 54 pairs of patients. The comparison of the critical variables after the matching confirmed no significant differences in OS between the selected patients (online suppl. Table 2). In these matched populations, the OS was similar in both groups (43.0 vs. 44.0 months, $p=0.878$ ).

\section{The Role of Surveillance in Cirrhotic Patients}

Among the 85 cirrhotic patients, 47 (55.3\%) received the diagnosis of ICC during a surveillance programme, whereas the remaining 38 (44.7\%) patients were diagnosed symptomatically or incidentally. These 2 groups were similar in terms of age and sex (Table 4 ). The 
Fig. 3. Comparison of OS between non-cirrhotic versus cirrhotic patients as a whole (a) and non-cirrhotic versus cirrhotic patients stratified according to their surveillance status (b). OS, overall survival.

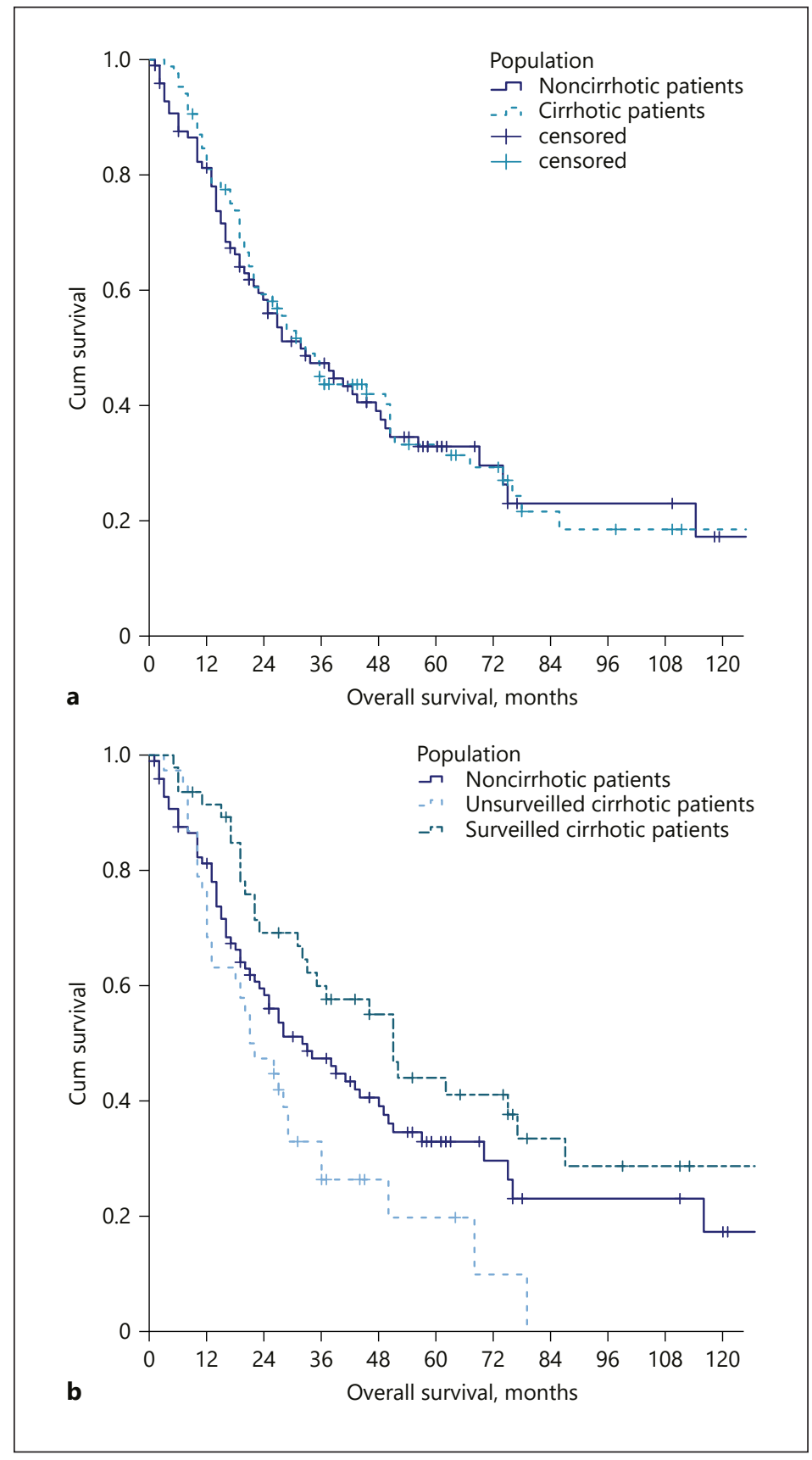

2 groups were also similar in terms of liver function and portal hypertension parameters (online suppl. Table 3). Instead, they differed in tumour dimensions (30 and $48 \mathrm{~mm}$ in surveyed and non-surveyed patients, respectively) $(p<0.001)$. As a result, surveyed patients were more likely to be treated surgically ( 59.8 vs. $28.9 \%$, respectively). Consequently, the crude survival was significantly different between these groups (51.0 vs. 21.0 months, $p=$ 0.001 ), with a crude reduction in the mortality risk of $59 \%$ in surveyed patients (HR $0.410-95 \%$ CI 0.238-0.705). Dissecting the cirrhotic patients according to their surveillance status and comparing them with non-cirrhotic patients revealed 3 different survival patterns (surveyed cirrhotic patients: median OS 51.0 months; non-cirrhotic patient: median OS 32.0 months; non-surveyed cirrhotic patients: median OS 21.0 months; $p=0.006$ ) (Fig. 3). 
Fig. 4. Comparison of OS between surveyed and non-surveyed cirrhotic patients following the adjustment for the lead-time bias. OS, overall survival. (c) 2020 The Author(s). Published by S. Karger AG, Basel www.karger.com/lic

Tovoli et al.: Intrahepatic Cholangiocarcinoma in Liver Cirrhosis

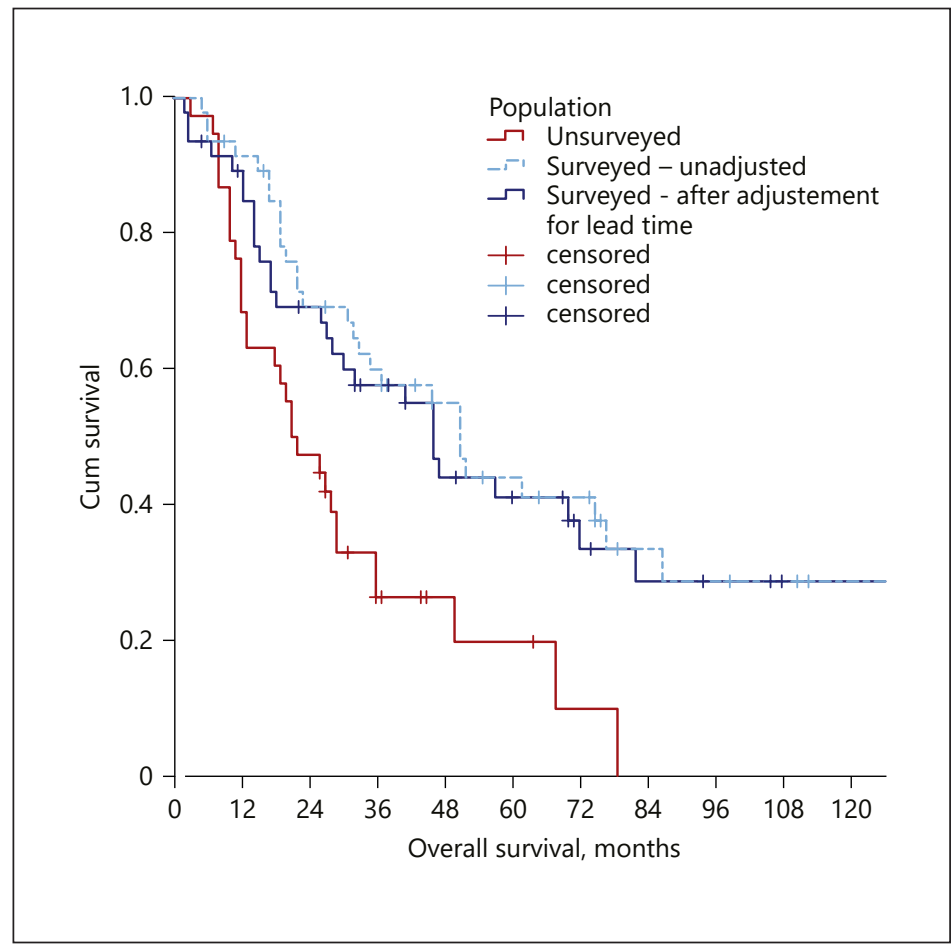

The correction for the lead-time bias left these results largely unchanged. In detail, the literature research identified a single pertinent paper [16], in which the median doubling time of ICC was 70 days. The median lead time in our cohort was 4.7 months (IQR 4.6-4.8). The adjusted median OS (46.2 months) was still higher in comparison with that of nonsurveyed patients $(p=0.004)$, with an adjusted reduction in the mortality risk of $54 \%$ (HR $0.464-95 \%$ CI 0.271-0.796) (Fig. 4).

\section{Additional Analyses}

To further assess the role of liver cirrhosis in determining the OS, we intended to re-analyse the survival after a stratification according to the surveillance status. A proper stratification was not possible since all of the non-cirrhotic patients had been diagnosed symptomatically, so a subgroup of non-cirrhotic surveyed patients could not be created. Indeed, 18 patients had chronic liver disease without liver cirrhosis (HBV: 2 patients, HCV: 8 patients, and alcoholic liver disease: 8 patients). In all of these cases, however, the underlying liver disease was discovered contextually to the ICC diagnosis. Viral hepatitis was diagnosed thanks to the pre-treatment standard investigation, while alcohol emerged as a risk factor when medical history data were collected.

Instead, a comparison between non-cirrothic patients and unsurveyed cirrhotic patients was feasible. When considering only these 2 populations, the univariable analysis confirmed tumour dimension, multinodularity, grading, performance status, metastatic disease, and treatment received as factors associated with the OS (cirrhosis reached a borderline significance $-p=0.060$ ). The multivariable Cox regression showed that only treatment was an independent predictor of OS (liver resection = reference; locoregional treatments HR 2.451 [95\% CI 1.930-4.516], $p=0.004$; chemotherapy HR 5.363 [95\% CI 3.026-9.317], $p<0.001$; best supportive care HR 14.627 [95\% CI 6.583-32.500], $p<0.001$ ). On the contrary, liver cirrhosis was not predictive of OS (HR 0.999 [95\% CI 0.534-1.869], $p=0.998$ ). 
Tovoli et al.: Intrahepatic Cholangiocarcinoma in Liver Cirrhosis

\section{Discussion}

So far, reports about the outcomes of ICC have provided conflicting results [17], especially in the cirrhotic population $[2-4,18]$, mirroring a large inter-centre variability. Consequently, we adopted a multicenter approach to reduce the risk of an inherent selection bias for the first time.

Our study population was mainly recruited in centres who routinely perform abdominal ultrasound examinations and most of which deliver clinical hepatological activities. This peculiarity allowed the recruitment of both patients who had been investigated to characterize focal lesions on healthy liver and cirrhotic patients performing ultrasound examinations during the surveillance programmes for HCC. For these reasons, the rate of cirrhotic patients was relatively high in comparison with previous series [4, 15]. Nonetheless, our whole cohort remained representative of the ICC population, as demonstrated by the outcomes of the surgical and non-surgical procedures and by the prognostic role of the AJCC-TNM classification, which is consistent with previous literature extents. By analysing our population, 2 main findings deserve discussion.

First, we found that cirrhotic patients with ICC were under a surveillance programme for HCC in about half of the cases. These patients had a significantly smaller ICC at the diagnosis and were more likely to be treated with surgical procedures. As a result, their prognosis was significantly better compared to that of non-surveyed cirrhotic patients. Even after applying the effects of a possible lead-time bias, the benefit of being under surveillance was largely confirmed. This is new evidence, as previous studies about ICC in cirrhotic patients never mentioned the surveillance status of the patients. A different possible rate of ICC diagnosed during surveillance might justify the conflicting evidence emerged in previous studies, which reported a higher, similar or lower OS in cirrhotic in comparison to non-cirrhotic patients.

Second, directly from the latter point, we found a similar OS in cirrhotic and non-cirrhotic ICC. This result is consistent with a previous report by Jesper and colleagues [4]. Differently from that study, however, we found differences in the clinical presentation of ICC in cirrhotic and non-cirrhotic patients. Overall, ICC was detected at an earlier stage in cirrhotic patients, with a lower likelihood of spread both to lymphnodes and to distant organs. As a result of these differences, we carried out a propensity score analysis to assess the role of cirrhosis as a prognostic factor correctly. Even after matching patients, liver cirrhosis did not result in a significantly lower OS. The presence of a large proportion of ICC diagnosed during surveillance is an element of critical importance in the interpretation of the aforementioned difference between cirrhotic patients as a whole and non-cirrhotic subjects. When performing a 3-group comparison, the OS significantly decreased from cirrhotic surveyed patients to non-cirrhotic and cirrhotic non-surveyed patients.

Our results have different implications in the clinical practice. First, liver cirrhosis itself should not be considered a contraindication to the treatment of ICC, as the outcome of cirrhotic patients does not differ from that of non-cirrhotic patients. When not contraindicated by liver function, portal hypertension or comorbidities, surgery should always be considered in first line in these patients, as it can dramatically improve their outcome. Similarly, locoregional and systemic treatments should not be discouraged, provided that liver function and coagulative parameters are permissive. As a second point, this study demonstrated for the first time that the semiannual ultrasound surveillance brings not only the known benefits for the early detection of HCC, but for ICC as well. While the prevalence of ICC alone would not justify the costs of the surveillance, this further benefit could be taken into consideration by policymakers and guidelines when evaluating the cost-effectiveness of the surveillance protocols in liver cirrhosis.

Our results should also be interpreted considering some limitations. First, the data of our study are retrospective in nature. However, cases were consecutively selected to reduce

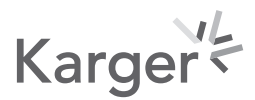


potential biases. Moreover, the risk of bias due to the presence of unbalanced prognosticators between groups was controlled applying a propensity score analysis. Second, our study included mostly patients in Child-Pugh A class. The small number of Child-Pugh B patients did not allow dedicated statistical analyses. As such, caution is needed before extending our findings to Child-Pugh B patients. Third, despite addressing the problem of the lead-time bias, other biases may be present when assessing the benefits of screening programmes, especially length-time bias. Length-time bias is defined as an overestimation of survival of screeningdetected cases caused by the relative excess of slower growing tumours detected with respect to symptomatic cases $[17,18]$. A precise estimation of the length-time bias, however, is still lacking in the setting of the early detection of ICC. Hence, dedicated modelling studies are welcome.

In conclusion, we found that cirrhotic patients might have a different clinical presentation and disease course of ICC according to their surveillance status, significantly influencing the chance of receiving curative surgery and therefore of obtaining satisfactory longterm outcomes. The discrepant results of previous studies evaluating the prognostic role of cirrhosis might be at least partly justified by the unknown rates of surveyed patients. In our series, cirrhosis (compensated in the majority of cases) was not associated with worse outcomes. Therefore, cirrhosis itself should not discourage either surgical, locoregional, or systemic treatments.

\section{Statement of Ethics}

The Ethics Committee approved the study (protocol 78/2017/O/OSSN)45/2016/U/Tess, 2016), which was conducted according to the ethical guidelines of the 1975 Declaration of Helsinki. Considering the retrospective design of the study and the unfavourable prognosis of the investigated disease, the Ethics Committee waived the need for informed consent for deceased patients and for patients whose clinical conditions had worsened to a point in which they were not able to sign a valid consent. All of the remaining patients provided a written informed consent for this study.

\section{Conflict of Interest Statement}

Francesco Tovoli: consultant for Bayer AG and advisory board for LaForce; Pietro Guerra: no conflicts to declare; Massimo Iavarone: speaker honoraria from Bayer, Gilead Science, Janssen, BTG, Abbvie, and MSD and consultant for BTG; Letizia Veronese: no conflicts to declare; Matteo Renzulli: no conflicts to declare; Stefania De Lorenzo: no conflicts to declare; Francesca Benevento: no conflicts to declare; Giovanni Brandi: advisory board for Eli-Lilly; Federico Stefanini: no conflicts to declare; Fabio Piscaglia: consultant for Astrazeneca, Bayer AG, EISAI, GE, and Tiziana life sciences; speaker's bureau honoraria from Bayer AG, Bracco, EISAI, and Laforce; and research contract with Esaote.

\section{Funding Sources}

This research did not receive any specific grant from funding agencies in the public, commercial, or notfor-profit sectors.

\section{Authors Contribution}

F.P. designed this study. All authors collected data. F.T. performed the statistical analysis and wrote the draft. All authors critically analysed the draft, reviewed literature, and approved the final version of the manuscript.

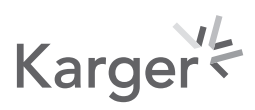




\begin{tabular}{l|l}
\hline Liver Cancer 2020;9:744-755 \\
\hline DOI: 10.1159/000509059 & $\begin{array}{l}\text { @ 2020 The Author(s). Published by S. Karger AG, Basel } \\
\text { www.karger.com/lic }\end{array}$ \\
\hline
\end{tabular}

Tovoli et al.: Intrahepatic Cholangiocarcinoma in Liver Cirrhosis

\section{References}

1 Ebata T, Ercolani G, Alvaro D, Ribero D, Di Tommaso L, Valle JW. Current status on cholangiocarcinoma and gallbladder cancer. Liver Cancer. 2016;6(1):59-65.

2 Li YY, Li H, Lv P, Liu G, Li XR, Tian BN, et al. Prognostic value of cirrhosis for intrahepatic cholangiocarcinoma after surgical treatment. J Gastrointest Surg. 2011;15(4):608-13.

3 Luo X, Yuan L, Wang Y, Ge R, Sun Y, Wei G. Survival outcomes and prognostic factors of surgical therapy for all potentially resectable intrahepatic cholangiocarcinoma: a large single-center cohort study. J Gastrointest Surg. 2014;18(3):562-72.

4 Jesper D, Heyn SG, Schellhaas B, Pfeifer L, Goertz RS, Zopf S, et al. Effects of liver cirrhosis and patient condition on clinical outcomes in intrahepatic cholangiocarcinoma: a retrospective analysis of 156 cases in a single center. Eur J Gastroenterol Hepatol. 2018;30(5):552-6.

5 Forner A, Vidili G, Rengo M, Bujanda L, Ponz-Sarvisé M, Lamarca A. Clinical presentation, diagnosis and staging of cholangiocarcinoma. Liver Int. 2019;39(Suppl 1):98-107.

6 Blachier M, Leleu H, Peck-Radosavljevic M, Valla DC, Roudot-Thoraval F. The burden of liver disease in Europe: a review of available epidemiological data. J Hepatol. 2013;58(3):593-608.

7 Shaib Y, El-Serag HB. The epidemiology of cholangiocarcinoma. Semin Liver Dis. 2004;24(2):115-25.

8 Banales JM, Cardinale V, Macias RIR, Andersen JB, Braconi C, Carpino G, et al. Cholangiocarcinoma: state-ofthe-art knowledge and challenges. Liver Int. 2019;39(Suppl 1):5-6.

9 Amin MB, Edge S, Greene F. AJCC cancer staging manual. 8th ed. Chicago, IL: Springer; 2017.

10 European Association for the Study of the Liver. EASL recommendations on treatment of hepatitis C 2018. J Hepatol. 2018;69(2):461-511.

11 Ghany MG, Morgan TR; AASLD-IDSA Hepatitis C Guidance Panel. Hepatitis C guidance 2019 update: American association for the study of liver diseases-infectious diseases society of America recommendations for testing, managing, and treating hepatitis C virus infection. Hepatology. 2020;71(2):686-721.

12 Walter SD, Stitt LW. Evaluating the survival of cancer cases detected by screening. Stat Med. 1987;6(8):885900.

13 Duffy SW, Nagtegaal ID, Wallis M, Cafferty FH, Houssami N, Warwick J, et al. Correcting for lead time and length bias in estimating the effect of screen detection on cancer survival. Am J Epidemiol. 2008;168(1):98-104.

14 Michaelson J, Satija S, Moore R, Weber G, Halpern E, Garland A, et al. Estimates of breast cancer growth rate and sojourn time from screening database information. J Women's Imaging. 2003;5(1):11-9.

15 Prevost TC, Launoy G, Duffy SW, Chen HH. Estimating sensitivity and sojourn time in screening for colorectal cancer: a comparison of statistical approaches. Am J Epidemiol. 1998;148(6):609-19.

16 De Rose AM, Cucchetti A, Clemente G, Ardito F, Giovannini I, Ercolani G, et al. Prognostic significance of tumor doubling time in mass-forming type cholangiocarcinoma. J Gastrointest Surg. 2013;17(4):739-47.

17 Ariizumi S, Yamamoto M. Intrahepatic cholangiocarcinoma and cholangiolocellular carcinoma in cirrhosis and chronic viral hepatitis. Surg Today. 2015;45(6):682-7.

18 Tyson GL, El-Serag HB. Risk factors for cholangiocarcinoma. Hepatology. 2011;54(1):173-84. 